\title{
Isolated oculomotor nerve palsy as a manifestation of diffuse large $B$ cell lymphoma: A case report
}

\author{
MUNTADHER M. KHALEEFAH ${ }^{1 *}$, SIDDHARTH NARAYANAN $^{2 *}$, HIBA A. AL DALLAL $^{3}$, \\ CHRISTOPHER M. JONES ${ }^{2}$, ROBERT P. FRIEDLAND ${ }^{1}$, ADRIANA E. PALADE ${ }^{1}$, \\ KERRI S. REMMEL ${ }^{1}$ and JIGNESH J. SHAH ${ }^{1}$ \\ Departments of ${ }^{1}$ Neurology, ${ }^{2}$ Surgery and ${ }^{3}$ Pathology and Laboratory Medicine, \\ University of Louisville Hospital, University of Louisville, Louisville, KY 40202, USA
}

Received March 17, 2020; Accepted August 7, 2020

DOI: $10.3892 / \mathrm{ol} .2020 .12147$

\begin{abstract}
An isolated third nerve palsy presenting as the primary manifestation of a lymphoma is rare, with only few cases having been described. The present study reports an unusual case of a healthy 67-year old male diagnosed with isolated right oculomotor nerve palsy (ONP), who was found to have an underlying B cell lymphoma. The patient's medical records were accessed upon consent. A thorough physical examination, including stroke and infections work-ups were performed. A chest computerized tomography (CT), brain magnetic resonance imaging and positron emission tomography (PET) scans and a mediastinal tissue biopsy, were performed as part of systematic diagnostic evaluations. The current report suggests that a PET fluorodeoxyglucose study or a CT scan of the chest, abdomen and pelvis (with contrast) may help in the early diagnosis of a cancer responsible for ONP, particularly if brain vessel imaging does not show a posterior cerebral artery aneurysm as a cause for the defect.
\end{abstract}

\section{Introduction}

Third cranial nerve dysfunction, oculomotor nerve palsy (ONP), can result from lesions anywhere along the nerve between the midbrain and the orbit (1). This can include the

Correspondence to: Dr Muntadher M. Khaleefah, Department of Neurology, University of Louisville, 500 South Preston Street, Louisville, KY 40202, USA

E-mail: muntadher.khaleefah@louisville.edu

${ }^{*}$ Contributed equally

Abbreviations: MRI, magnetic resonance imaging; CSF, cerebrospinal fluid; PET, positron emission tomography; CNS, central nervous system; ONP, oculomotor nerve palsy; DLBCL, diffuse large B cell lymphoma; HIV, human immunodeficiency virus; IHC, immunohistochemistry; FISH, fluorescence in situ hybridization

Key words: oculomotor, cranial nerve, MRI, lymphoma, palsy, $\mathrm{CSF}, \mathrm{CT}$ oculomotor nucleus, the fascicles in the midbrain tegmentum and the spaces it passes through, including the subarachnoid space, the cavernous sinus and the superior orbital fissure (1). The major causes for ONP include congenital factors, trauma, migraine, aneurysm, diabetes, microvascular ischemia and inflammation $(1,2)$, with neoplasms and tumors being less common (2).

Cranial nerves and their surrounding leptomeninges and cavernous sinus are often known to be involved in lymphomas (3). ONP as a presenting feature of an underlying benign or malignant tumor is rare. However, its occurrence as the first presenting manifestation of a lymphoma is particularly unusual, especially when no other lymphoma manifestations have initially been identified (3). We describe a rare case of a 67-year old man who presented with an isolated ONP as the primary manifestation of a diffuse large B cell lymphoma (DLBCL), exhibiting without palpated masses, enlarged lymph nodes or peripheral blood abnormalities.

\section{Case report}

A 67-year old right-handed Caucasian male with no significant past medical history developed a sudden, continuous sharp right-sided retro-orbital pain, 8/10 in intensity. He denied having photophobia, phonophobia, blurry vision, nausea or vomiting. Upon an outside hospital admit (in-patient), he was found to have an elevated serum C-reactive protein levels, and he underwent a temporal artery (TA) biopsy and was prescribed steroids (prednisone, $60 \mathrm{mg} /$ day with taper over 1 month). The patient was discharged with a working diagnosis of temporal arteritis. Once the TA biopsy came back as negative, the steroids were stopped.

A month later, he returned to the same hospital with a right eye ptosis. A computed tomography angiography of the head and neck and magnetic resonance imaging (MRI) of the brain were unremarkable. Upon transfer to our University of Louisville hospital for further work-up, the patient revealed that his previous retro-orbital pain had improved but that now he was now experiencing double vision. Notably, his family history revealed a very strong disposition for cancer (father died from lymphoma, two sisters passed away from breast cancer and a brother died of testicular cancer). 
Upon physical examination he had right upper eyelid ptosis, diplopia, right dilated pupil (not reactive to light), was not able to move the right eye medially or upward and he denied having any periorbital pain. A dilated ophthalmoscopic exam was unremarkable, including the remainder of the neurological exams, such as assessing for motor/sensory and other cranial nerves function, gait and co-ordination examinations, which were normal. A stroke assessment (including a brain MRI and CT angiogram of the head and neck images, and blood tests) was unremarkable. Evaluation for infections [including human immunodeficiency virus (HIV) and rapid plasma regain test for syphilis] were also negative. The patient was diagnosed having an ONP, prescribed aspirin (325 mg, daily) and atorvastatin (80 mg, daily) and discharged from the stroke service.

Later on, the same day, the patient developed B symptoms, including chest pain, sweating and shortness of breath accompanied by double vision and fever, and was readmitted to the hospital. The electrocardiogram was unremarkable. A coincidental chest CT (pulmonary embolism protocol) showed a large posterior mediastinal mass extending from the level of the carina into the upper abdomen behind the crura of the diaphragm. The mass completely encased the esophagus and aorta, measuring $\sim 16.7 \mathrm{~cm}$ in craniocaudal dimension. It had a maximum size on the axial images of $\sim 11.8 \times 14.1 \mathrm{~cm}$ (Fig. 1). Staging and prognostic evaluation of the mass revealed a stage IV DLBCL with a revised international prognostic index (R-IPI) score of 4.

Formalin-fixed $(10 \%, 24 \mathrm{~h}$ at room temperature), paraffin-embedded mediastinal biopsy sections were cut into $4 \mu \mathrm{m}$ thickness sections, deparaffinized, and stained with hematoxylin and eosin (H\&E) and immunohistochemistry (IHC) markers as per manufacturers' instructions. Briefly, after deparaffinization, sections were washed with ethanol gradient $(100,95,75$ and $50 \%$ for $5 \mathrm{~min}$ each) then rehydrated and treated with $3 \% \mathrm{H}_{2} \mathrm{O}_{2}$ for $5 \mathrm{~min}$ at room temperature to block endogenous peroxidase activity. Slides were steamed in retrieval solution (citrate buffer, $\mathrm{pH} 6$ or Tris/EDTA buffer, $\mathrm{pH} 9$ ) for $15 \mathrm{~min}$ and then cooled for $15 \mathrm{~min}$, and blocked in 5\% FBS (Jackson ImmunoResearch Laboratories, Inc.) that was diluted in wash buffer with $1 \%$ BSA at room temperature for $15 \mathrm{~min}$. For IHC, the following antibodies were used: CD20 (1:150; cat. no. ab78237; Abcam), CD10 (1:300; cat. no. ab256494; Abcam), BCL6 (1:500; cat. no. ab172610; Abcam), MUM1 (1:200; cat. no. ab133590; Abcam),Ki-67 (1:50; cat. no. MIB-1; Labvision), BCL2 (1:300; cat. no. ab32124; Abcam) and c-MYC (5 $\mu \mathrm{g} / \mathrm{ml}$; cat. no. ab32072; Abcam) at $4^{\circ} \mathrm{C}$ overnight. After being washed three times with PBS, the sections were stained with goat anti-rabbit secondary antibody (1:500; cat. no. ab150077; Abcam) for $30 \mathrm{~min}$ at $37^{\circ} \mathrm{C}$. After applying 3,3'diaminobenzidine (DAB) for color development at room temperature for $5 \mathrm{~min}$, the sections were subsequently counterstained with hematoxylin. Each slide was individually reviewed and scored by two experienced pathologists using light microscopy.

The tumor cells were positive for CD20, B cell-specific activator protein, CD10 (cell surface marker), B cell lymphoma (BCL)-6 [>50\%, positive cells (from a total of 200) were counted from three different areas of the slide and averaged to get the percentage], but had a low expression for multiple myeloma 1 (MUM1, <5\%; Fig. 2). These results categorized

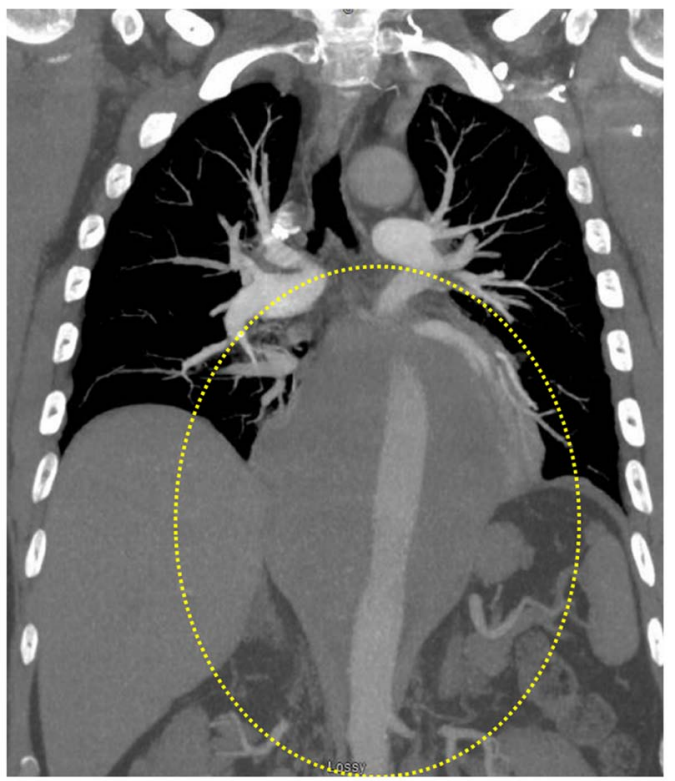

Figure 1. CTA chest image. A chest CTA revealed a large posterior mediastinal mass extending from about the level of the carina into the upper abdomen behind the crura of the diaphragm (as highlighted by the yellow dotted circle). CTA, computed tomography angiography.

this large B cell lymphoma as a germinal-center phenotype. The Ki-67 (proliferative marker) staining showed high nuclear labeling (80\%) (Fig. 2I) indicating a highly proliferative aggressive lymphoma. The tumor cells were diffusely positive for BCL2 expression (>50\%) (Fig. 2F) but showed very low expression for c-MYC (Fig. 2H), suggesting a possible BCL2 rearrangement that was later confirmed by fluorescent in situ hybridization (FISH; performed by NeoGenomics Laboratories). However, no c-MYC or BCL6 rearrangements were detected. All IHC controls [using normal tonsil tissue having both, positively stained (lymphoid tissue) and the negatively stained (squamous mucosa) areas] showed specific staining patterns (Fig. 3). The concurrent flow cytometric analysis (performed at the Department of Pathology, University of Louisville) detected an abnormal CD10 (+) monoclonal large B cell population.

Apart from slightly elevated total protein levels, the lumbar puncture was otherwise normal. The cerebrospinal fluid (CSF) culture was negative for any microorganisms (no growth for five consecutive days), and the cytology identified no malignant cells. A brain MRI (with and without contrast) did not show any leptomeningeal enhancement. An aggressive chemotherapy treatment was followed wherein the patient received six cycles of etoposide phosphate, prednisone, oncovin (vincristine sulfate), cyclophosphamide, hydroxydaunorubicin (doxorubicin hydrochloride) with rituximab and pegfilgrastim, and two cycles of high-dose intrathecal methotrexate. Despite this, the ONP did not resolve. A positron emission tomography (PET) scan after chemotherapy completion showed new areas of lymphoma spread including the ribs and mediastinum (Fig. 4). The patient developed back pain and an MRI of the lumbar spine showed diffuse leptomeningeal enhancement, suspicious for the spread of his lymphoma (Fig. 5). Based on the poor prognosis, the patient opted for palliative care and later passed away. 

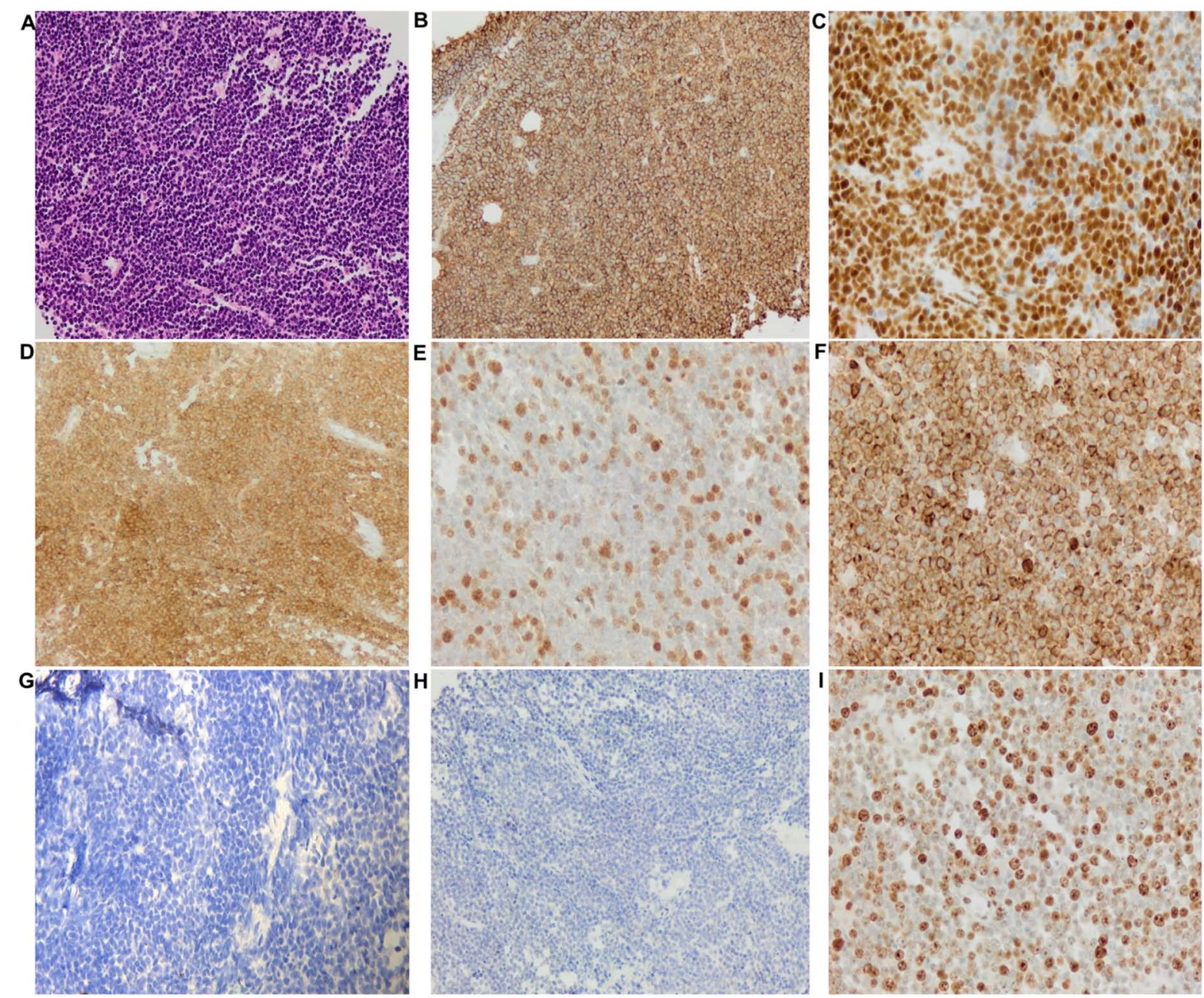

Figure 2. Histopathological images of the mediastinal biopsy specimen. (A) Large numbers of lymphocytes, medium to large, with oval or round nuclei containing fine chromatin and scanty cytoplasm (hematoxyclin and eosin staining, x20 magnification). Immunohistochemical staining (compared with appropriate controls) revealed strong, positive expression for (B) CD20 (membranous, diffuse), (C) BSAP (nuclear, partial), (D) CD10 (membranous, diffuse), (E) BCL6 (nuclear), and (F) BCL2 (membranous). Tissue sections showed low expression for (G) multiple myeloma 1 (no nuclear staining) and (H) MYC. (I) High expression of proliferative marker Ki-67 (80\%).

\section{Discussion}

An isolated ONP occurring as a neurological manifestation of an underlying lymphoma is rare with only a few cases having been reported $(1,2)$. The present case was peculiar as, apart from an isolated ONP finding, no other physical examination or laboratory results indicated lymphoma. Usually, patients with lymphoma display other signs, such as increased leucocyte count, B symptoms and the development of mediastinal masses (3). The latter was only identified from a coincidental $\mathrm{CT}$ chest in the present patient, at a later stage. Retrospectively, it is also interesting to note that the patient had gradual weight loss which could have been another manifestation of the underlying lymphoma, but it went unrecognized. Cases presenting with ONP due to lymphomas and sarcomas have been previously summarized $(4,5)$. A summary of new cases reported between 2011 and 2019 are presented in Table I. Out of the 12 lymphoma cases identified, seven had DLBCL, three had Burkitt lymphoma, one had Hodgkin lymphoma and one had non-Hodgkin lymphoma.

A tumor can associate with the cranial nerves locally, via direct infiltration or by a paraneoplastic process (6). Cranial nerve involvement depends on its anatomical course and the site of the tumor (1). The most common reason suggested for the occurrence of third nerve palsy is the invasion of the cavernous sinus and the surrounding leptomeninges with or without oculomotor nerve infiltration $(4,5,7)$. In the current case, the MRI brain and orbit (with and without contrast) did not show any pathology. A possible reason for this could be that the patient received steroids (for a suspected TA) prior to the brain MRI, which may have resulted in a false negative image and thus masking signs of infiltration or a leptomeningeal enhancement. As the patient had pain in the same eye before starting steroids, it would have been highly probable that signs of infiltration would have been identified with a cranial MRI, as this would have justified the pain and may contributed to an earlier diagnosis of the lymphoma. Notably, cranial nerve palsies have been reported even in the presence of normal MRIs $(5,6)$. Intravascular lymphoma, one of the DLBCL subtypes, has a high frequency of nerve infiltration including the CNS (4), but unfortunately no biopsy was performed in the present case to confirm this possibility. Lymphatic micro-infiltration seemed an unlikely cause, due to a negative CSF, making paraneoplastic process as another 


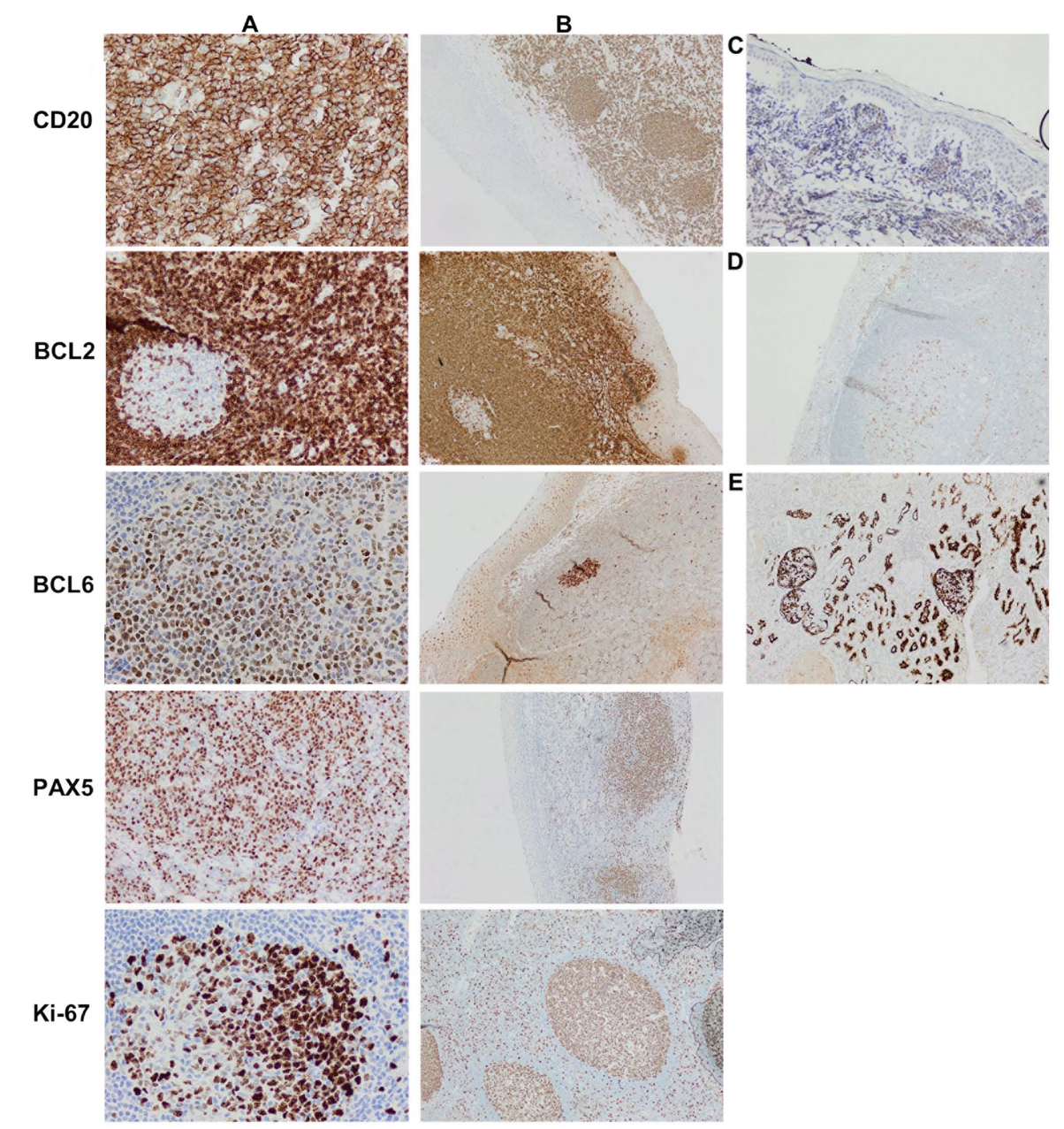

Figure 3. Immunohistochemical control images. Normal tonsil tissue was used as a control showing both (A) positively stained lymphoid tissue and the (B) absence of staining for squamous mucosa. (C) Normal tonsil tissue showing strong MYC expression having $>10 \%$ MYC positive lymphoid areas, with a weak, distinct staining of the mantle zone B-cells ( 10\%). (D) Normal tonsil tissue with MUM1 staining. (E) Normal renal proximal tubule epithelial cells stained with CD10 showing a strong, predominantly membranous and cytoplasmic staining; distal tubules showing absence of staining. MUM1, multiple myeloma 1. Magnifications: Panel A, C, D, x400; panel B, x200.

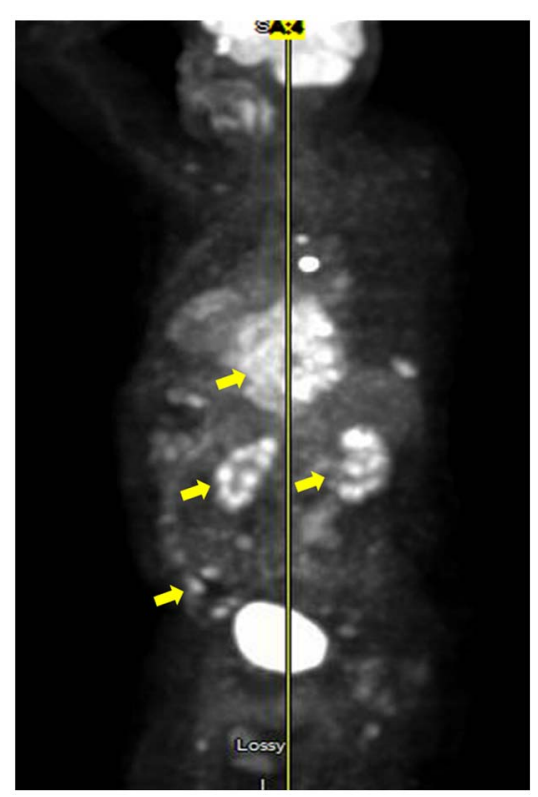

Figure 4. A positron emission tomography scan showing the hyper-metabolic tissue of the lymphoma affecting the posterior mediastinum, bilateral kidneys and some bone marrow indicating disseminated disease (as highlighted by the yellow arrows).

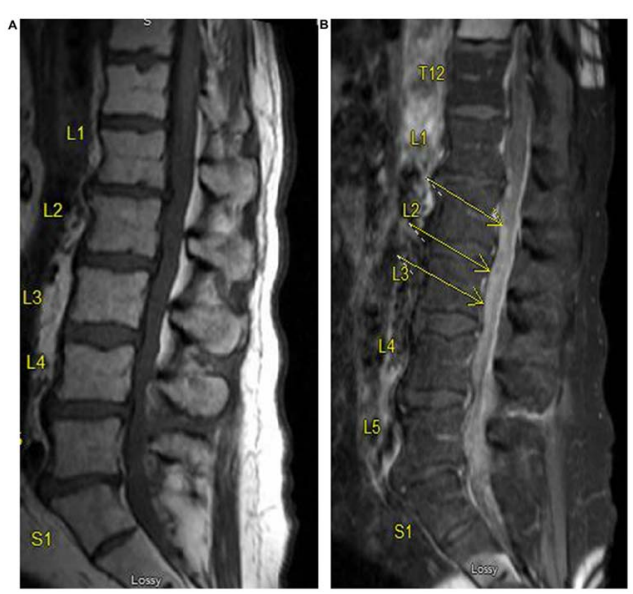

Figure 5. An magnetic resonance imaging of the L spine. (A) T1 without contrast and, (B) T1 with contrast showing diffuse leptomeningeal enhancement suspicious for the lymphoma spread.

possible mechanism of how the tumor came to affect the third cranial nerve. This process may precede the actual lymphocytic infiltration by weeks to months (1). Paraneoplastic 


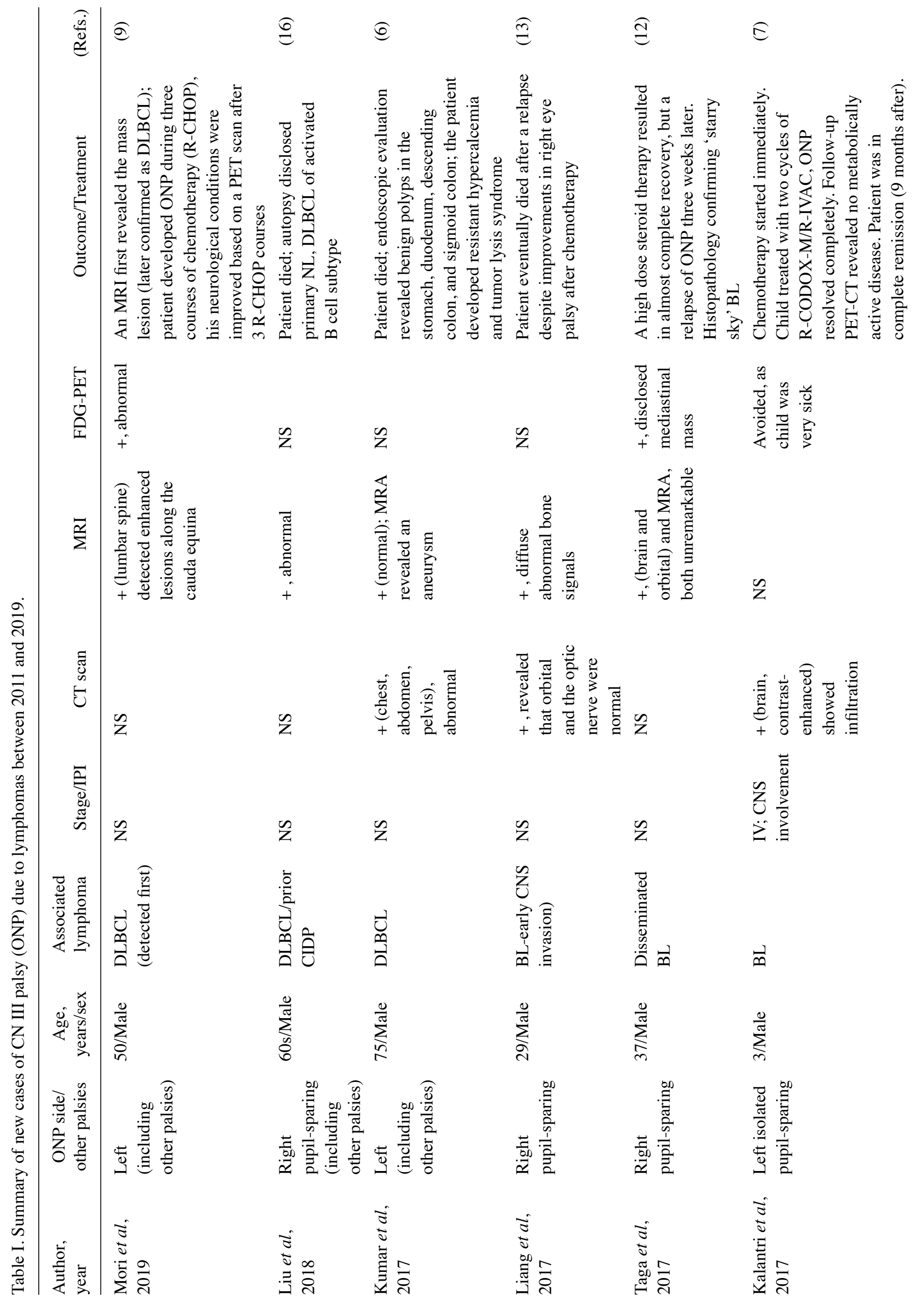




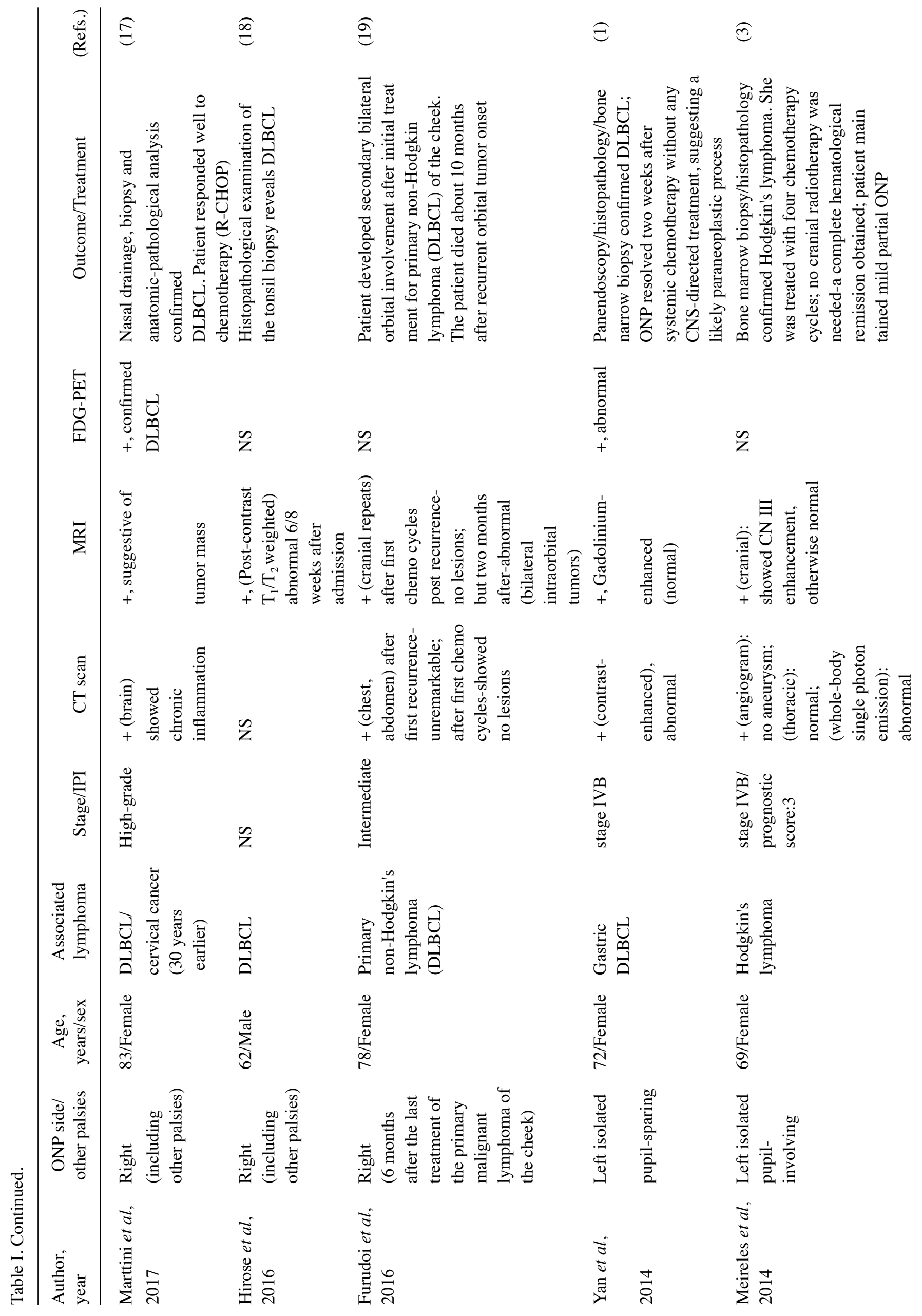


syndromes are a class of heterogeneous disorders with diverse presentations caused by an immune response to an underlying malignancy (6). While rarely associated with Hodgkin's and non-Hodgkin's lymphoma, these often develop into lymphoma during the end stages of the disease (6). However, early detection of asymptomatic gall bladder cancer in a patient with multiple cranial palsies during possible paraneoplastic neurological syndrome has also been reported (8).

In some of the previously patients (Table I), including the present patient, the pupil was involved (right dilated pupil). Previous reports have shown a direct infiltration of the oculomotor nerve upon histological or MRI examination, suggesting that this is a major pathological mechanism of pupil involvement (4,5). MRI and PET scans are the imaging modalities of choice for evaluation of patients with lymphoma and suspected neural involvement (9). In a report reviewing 14 lymphoma cases with ONP, ten were assessed using brain MRIs and eight of these patients had CNS involvement (5). CSF analysis and brain MRIs may not always yield positive results, and repeat testing may be essential for improving chances of identification $(5,7)$, which was also true for the present case as only during a repeat MRI of the lumbar spine was the lymphoma identified.

DLBCL is the most common of the aggressive non-Hodgkin's lymphoma in the United States of America (10). Based on the cell of origin, it is categorized into three different subgroups: Germinal center B cell-like; activated C cell-like and unclassifiable (11). Accuracy of classification has become increasingly sophisticated as it appears to have prognostic value (in terms of overall survival) and bears strong significance in making treatment decisions (11). Different immunohistochemical (IHC) algorithms, such as the Hans algorithm, have been proposed in the last decade to classify DLBCL subgroups (11). This IHC classification and molecular studies (Figs. 2 and 3) were used to confirm the diagnosis of DLBCL in our patient.

New reports have identified an isolated ONP as a neurological manifestation for Burkitt's lymphoma $(7,12,13)$. Distinguishing between Burkitt lymphoma and DLBCL was critical, as the two diseases require different management (14). Almost all forms of Burkitt's lymphoma are identified with a $M Y C$ locus rearrangement (translocation at $8 \mathrm{q} 24$ ) that results in increased MYC protein levels, promoting cell proliferation (11). The Ki-67 staining pattern in such cases is usually $>95 \%$ (15). In the current case, the Ki-67 staining pattern (80\%) together with the IHC and FISH data confirmed the absence of MYC involvement, thus reducing the likelihood of the tumor being ascribed as a Burkitt's lymphoma. In addition, the patient tested negative for HIV thus further reducing the possibility of the ONP presenting as a Burkitt lymphoma manifestation instead of a DLBCL. A recent report identified only three cases of ONP as the Burkitt manifestation, occurring in patients with HIV infection (12). Burkitt's is rapidly fatal if left untreated but curable with intensive chemotherapy (doxorubicin, alkylators, vincristine and etoposide); however, it cannot be cured by the protocol used for DLBCL (15). Diagnostic accuracy is therefore essential to prevent mistreatment.

Cranial neuropathies are known to be associated with lymphomas, but as observed in the present report, occurrence of an isolated ONP can be considered as a potential differential 
for an underlying DLBCL. The current report suggests that ordering a PET scan or a chest, abdomen and pelvis CT (with contrast) may permit an early diagnosis of this cancer in patients with unexplained ONP, particularly if brain vessel imaging does not show a posterior cerebral artery aneurysm as a cause.

\section{Acknowledgements}

Not applicable.

\section{Funding}

No funding was received.

\section{Availability of data and materials}

The datasets used and/or analyzed during the current study are available from the corresponding author on reasonable request.

\section{Authors' contributions}

MMK collected the data. SN, HAAD and MMK analyzed the literature and wrote the manuscript. CMJ, AEP, KSR, RPF and JJS reviewed and edited the manuscript. All authors read and approved the final manuscript.

\section{Ethics approval and consent to participate}

The University of Louisville Institutional Review Board approved the present study (IRB no. 20.0131; approval no. 702074).

\section{Patient consent for publication}

The informed consent was obtained from next of kin, including consent to publish the case study.

\section{Competing interests}

The authors declare that they have no competing interests.

\section{References}

1. Yan SY, Peng YJ, Lin CS, Peng GS and Chang PY: Isolated oculomotor nerve palsy as a paraneoplastic manifestation of gastric diffuse large B-cell lymphoma: A case report. Oncol Lett 8: 1983-1985, 2014.

2. Kim T, Nam K and Kwon BS: Isolated oculomotor nerve palsy in mild traumatic brain injury. Am J Phys Med Rehabil 99: $430-435,2020$

3. Meireles J, Garrett MC and Abreu P: Isolated III cranial nerve palsy: A Hodgkin's lymphoma? BMJ Case Rep 2014 bcr2014203999, 2014.
4. Bhatti MT, Schmalfuss IM and Eskin TA: Isolated cranial nerve III palsy as the presenting manifestation of HIV-related large B-cell lymphoma: Clinical, radiological and postmortem observations: Report of a case and review of the literature. Surv Ophthalmol 50: 598-606, 2005.

5. Sato H, Hashimoto T, Yoneda S, Hirabayashi K, Oguchi K and Higuchi K: Lymphoma as a cause of isolated oculomotor nerve palsy. J Clin Neurosci 18: 1256-1258, 2011.

6. Kumar K, Ahmed R, Bajantri B, Singh A, Abbas H, Dejesus E, Khan RR, Niazi $M$ and Chilimuri S: Tumors presenting as multiple cranial nerve palsies. Case Rep Neurol 9: 54-61, 2017.

7. Kalantri SA, Nayak A, Datta S and Bhattacharyya M: Isolated third nerve palsy: A rare neurological presentation of Burkitt's lymphoma. BMJ Case Rep 2017: bcr2017219670, 2017.

8. Kaido M, Yuasa Y, Yamamoto T, Munakata S, Tagawa N and Tanaka K: A case of possible paraneoplastic neurological syndrome presenting as multiple cranial nerve palsies associated with gallbladder cancer. Rinsho Shinkeigaku 56: 617-621, 2016 (In Japanese).

9. Mori Y, Yamamoto K, Ohno A, Fukunaga M and Nishikawa A: Primary central nervous system lymphoma with peripheral nerve involvement: Case report. Cureus 11: e5675, 2019.

10. Friedberg JW and Fisher RI: Diffuse large B-cell lymphoma. Hematol Oncol Clin North Am 22: 941-952, ix, 2008.

11. Swerdlow SH: Diagnosis of 'double hit' diffuse large B-cell lymphoma and B-cell lymphoma, unclassifiable, with features intermediate between DLBCL and Burkitt lymphoma: When and how, FISH versus IHC. Hematology Am Soc Hematol Educ Program 2014: 90-99, 2014.

12. Taga A, Russo M, Florindo I and Pavesi G: Isolated third cranial nerve palsy leading to the diagnosis of disseminated Burkitt lymphoma: A case report and literature review. Neurologist 22: 182-185, 2017.

13. Liang Y, Ding L, Li X, Wang W and Zhang X: Oculomotor nerve palsy as a preceding symptom of adult sporadic Burkitt lymphoma: A case report and review of the literature. Oncol Lett 13: 1315-1318, 2017.

14. Bellan C, Stefano L, Giulia de F, Rogena EA and Lorenzo L: Burkitt lymphoma versus diffuse large B-cell lymphoma: A practical approach. Hematol Oncol 28: 53-56, 2010.

15. Kalisz K, Alessandrino F, Beck R, Smith D, Kikano E, Ramaiya NH and Tirumani SH: An update on Burkitt lymphoma: A review of pathogenesis and multimodality imaging assessment of disease presentation, treatment response, and recurrence. Insights Imaging 10: 56, 2019.

16. Liu KC, Hennessey MA, McCall CM and Proia AD: Ocular involvement in neurolymphomatosis. Am J Ophthalmol Case Rep 10: 148-151, 2018.

17. Marttini Abarca JDP, Portilla Franco ME and Pastor Vicente E: A Third nerve palsy that reveals a diffuse large B cell lymphoma in an octogenarian patient. Rev Esp Geriatr Gerontol 52: 286-288, 2017 (In Spanish).

18. Hirose T, Nakajima H, Shigekiyo T, Yokote T, Ishida S and Kimura F: Malignant lymphoma presented as recurrent multiple cranial nerve palsy after spontaneous regression of oculomotor nerve palsy: A case report. Rinsho Shinkeigaku 56: 48-50, 2016 (In Japanese).

19. Furudoi S, Yoshii T and Komori T: Secondary bilateral orbital involvement from primary non-hodgkin lymphoma of the cheek. Kobe J Med Sci 62: E55-E57, 2016.

20. Tsai CH, Lee EJ, Liu YS, Chen YC, Shih YH and Chuang MT: Isolated oculomotor nerve palsy caused by diffuse large B cell lymphoma. Acta Neurol Belg 113: 103-104, 2013.

This work is licensed under a Creative Commons Attribution-NonCommercial-NoDerivatives 4.0 International (CC BY-NC-ND 4.0) License. 